\title{
ON THE CUSPIDAL SPECTRUM OF THE ARITHMETIC HECKE GROUPS
}

\author{
MARVIN I. KNOPP
}

D.H. Lehmer in memoriam

\begin{abstract}
Let $\Gamma(\sqrt{m})(m=1,2,3)$ be the three arithmetic Hecke groups, generated by the translation $z \mapsto z+\sqrt{m}$ and the inversion $z \mapsto-\frac{1}{z} .(\Gamma(1)$ is the modular group $\operatorname{PSL}(2, Z)$.) The purpose of this article is to present several theoretical results on $\Sigma(\sqrt{m})$, the weight-zero cuspidal spectrum of $\Gamma(\sqrt{m})$, which are of interest from a computational perspective, since they have application to the numerical study both of the spectra themselves and the Fourier coefficients of the associated Maass wave forms. The first of these is the theorem that $\Sigma(1) \subset \Sigma(\sqrt{m})$ for $m=2$ and $m=3$. Additional results explicate the action of the Hecke operators, for the three groups in question, upon Maass wave forms, in particular upon their Fourier coefficients. These results are motivated in part by work of Stark-and more recent work of Hejhal and Hejhal and Arno-which demonstrates the importance of the Hecke operators for the numerical study of the Fourier coefficients of Maass wave forms on $\Gamma(1)$.
\end{abstract}

\section{INTRODUCTION}

The spectral theory of discrete groups of linear fractional transformations acting on the upper half-plane $\mathscr{H}$ (or, what is the same, any half-plane or disc) has received much attention since the seminal work of Maass [6, 7] and Selberg $[10,11]$. Some of this has focused upon numerical calculation of the cuspidal spectrum $\Sigma(1)$ of the modular group $\Gamma(1)$ [4, 14]. Quite recently, several mathematicians have initiated numerical studies of the cuspidal spectra $\Sigma(\sqrt{m})$ of the Hecke groups $\Gamma(\sqrt{m}), m=2$ and $3[3,14]$. (These two groups are commensurable with $\Gamma(1)$, the only Hecke groups-aside from $\Gamma(1)$ itself-to have this property.)

The purpose of this article is to present several theoretical results on $\Sigma(1)$, $\Sigma(\sqrt{2})$, and $\Sigma(\sqrt{3})$ which have interest from a computational perspective, since they can find application to the numerical computation both of the spectra and the Fourier coefficients of the associated Maass wave forms. (See $\S 2$ for definitions.) The first of these results is the observation that the cuspidal spectrum of $\Gamma(1)$ is contained in that of $\Gamma(\sqrt{m})$, for $m=2$ and $m=3$. (See $\S 2$, Theorem 1.) This result shows that the calculations of [14] are flawed. (In $\S 2, m$ will

Received by the editor July 27, 1992.

1991 Mathematics Subject Classification. Primary 11F72, 11 F25.

Research supported in part by grants from the National Science Foundation and the National Security Agency. 
have the three values $m=1,2,3$; elsewhere $m$ has the two values $m=2$ and $m=3$.)

Additional results serve to explicate the action of the Hecke operator-not only for $\Gamma(1)$, but for $\Gamma(\sqrt{2})$ and $\Gamma(\sqrt{3})$ as well-upon the Maass wave forms, in particular upon their Fourier coefficients $(\S \S 5-6)$. This discussion is motivated by work of Stark [13] and more recent work of Hejhal [3] and Hejhal and Arno [5], which demonstrates clearly the importance of the Hecke operators for the numerical study of the Fourier coefficients of the Maass wave forms on $\Gamma(1)$. Here we emphasize especially the little-known Hecke operators on $\Gamma(\sqrt{m})$, introduced in [1], since these can be applied in the same way to the numerical study of the Fourier coefficients of Maass wave forms on $\Gamma(\sqrt{m})$. The existence of the Hecke operators on $\Gamma(\sqrt{m})(m=2,3)$ is best understood as a consequence of the fact (following from a straightforward calculation) that a subgroup of index 2 in $\Gamma(\sqrt{m})$ is conjugate to $\Gamma_{0}(m)$ under the inversion $z \mapsto-1 / \sqrt{m} z$. The Hecke operators for $\Gamma(\sqrt{m})$ derive from the well-known Hecke operators for $\Gamma_{0}(m)$.

\section{Preliminaries}

Let

$$
S_{m}=\left(\begin{array}{cc}
1 & \sqrt{m} \\
0 & 1
\end{array}\right), \quad T=\left(\begin{array}{rr}
0 & -1 \\
1 & 0
\end{array}\right) .
$$

The three groups we consider here are the arithmetic Hecke groups

$$
\Gamma(\sqrt{m})=\left\langle S_{m}, T\right\rangle, \quad m=1,2,3,
$$

considered as groups of linear fractional transformations. The relations in $\Gamma(\sqrt{m})$ are $T^{2}=\left(S_{m} T\right)^{\alpha(m)}=I$, with $\alpha(1)=3, \alpha(2)=4, \alpha(3)=6$.

Let $m=1,2$ or 3 . A sufficiently smooth function $f \not \equiv 0$ defined on the upper half-plane $\mathscr{H}$ is called a Maass wave form on $\Gamma(\sqrt{m})$, with eigenvalue $\lambda$, if

(i) $\quad f(z+\sqrt{m})=f(z), \quad f(-1 / z)=f(z)$;

(ii) $\quad-y^{2}\left(f_{x x}+f_{y y}\right)=\lambda f, \quad z=x+i y$;

(iii) $\quad f(x+i y) \rightarrow 0$ exponentially, as $y \rightarrow+\infty$.

We call $\lambda$ an eigenvalue of the hyperbolic Laplace operator $\Delta$, defined by

$$
(\Delta f)(z)=-y^{2}\left(f_{x x}(z)+f_{y y}(z)\right), \quad z=x+i y .
$$

The cuspidal spectrum $\Sigma(\sqrt{m})$, of $\Gamma(\sqrt{m})$, is defined to be the set of those $\lambda$ occurring as eigenvalues associated with Maass wave forms on $\Gamma(\sqrt{m})$.

If a Maass wave form $f$ satisfies $f(-\bar{z})=f(z)$, then $f$ is called even; if instead $f$ satisfies $f(-\bar{z})=-f(z)$, it is called odd.

Remark. If a Maass wave form on $\Gamma(\sqrt{m})$ with associated eigenvalue $\lambda$ were neither even nor odd, then we could form the nontrivial functions

$$
f_{e}(z)=f(z)+f(-\bar{z}), \quad f_{o}(z)=f(z)-f(-\bar{z}),
$$

both of which are again Maass wave forms with eigenvalue $\lambda$. Here, $f_{e}$ is even and $f_{o}$ is odd, so that $f_{e}$ and $f_{o}$ are linearly independent. This would contradict the widely-held belief that the space of Maass wave forms associated with a fixed eigenvalue $\lambda$ has dimension one. 
Let $\Sigma_{e}(\sqrt{m})$ denote the subset of $\Sigma(\sqrt{m})$ consisting of eigenvalues associated with even Maass wave forms. Similarly, $\Sigma_{o}(\sqrt{m})$ is the set of eigenvalues arising from odd Maass wave forms. Then by the remark above,

$$
\Sigma(\sqrt{m})=\Sigma_{e}(\sqrt{m}) \cup \Sigma_{o}(\sqrt{m}) .
$$

We prove a slightly strengthened version of the result ennunciated in the introduction.

Theorem 1. There holds $\Sigma_{e}(1) \subset \Sigma_{e}(\sqrt{m})$ and $\Sigma_{o}(1) \subset \Sigma_{o}(\sqrt{m})$, for $m=2$ and $m=3$.

\section{THE DIRECT OPERATOR}

The proof of Theorem 1 depends only upon a straightforward application of the operator $\Phi_{m}$ defined by

$$
\Phi_{m}[f(z)]=f(z / \sqrt{m})+f(\sqrt{m} z) .
$$

Hecke [2] observed that if $f$ is invariant with respect to $\Gamma(1)$, then $\Phi_{m} f$ is invariant with respect to $\Gamma(\sqrt{m})$. (Hejhal [3] points out that Rausenberger [8] noticed this fifty years earlier.) In fact, much more is true concerning $\Phi_{m}$; these further properties of $\Phi_{m}$ are essential to the proof of Theorem 1, which follows-almost directly-from them. They are stated in

Proposition 2. If $f$ is a Maass wave form on $\Gamma(1)$ with eigenvalue $\lambda$, then $\Phi_{m} f$ is a Maass wave form on $\Gamma(\sqrt{m})$ with eigenvalue $\lambda$. If $f$ is even (odd), then $\Phi_{m} f$ is even (odd).

The proof of this amounts to nothing more than the obvious verifications. To complete the proof of Theorem 1, then, it suffices to prove

Lemma 3. If $f$ satisfies condition (1) (iii) and $\Phi_{m} f \equiv 0$, then $f \equiv 0$.

Proof. $\Phi_{m} f \equiv 0$ implies that $f(z)=-f(m z)$ for $z$ in $\mathscr{H}$. By iteration, it follows that, for each $z$ in $\mathscr{H}, f(z)=(-1)^{k} f\left(m^{k} z\right)$, with $k$ a positive integer. Letting $k \rightarrow+\infty$ and using condition (1) (iii), we conclude that $f(z)=0$ for each $z$ in $\mathscr{H}$. This completes the proof of Lemma 3, hence of Theorem 1.

\section{THE INVERSE OPERATOR}

Analogous to $\Phi_{m}$ is the operator $\Psi_{m}$, first defined in [1]. This is given by

$$
\Psi_{m}[\varphi(z)]=\varphi(\sqrt{m} z)+\sum_{j=0}^{m-1} \varphi\left(\frac{z+j}{\sqrt{m}}\right) .
$$

A calculation shows that if $\varphi$ is invariant with respect to $\Gamma(\sqrt{m})$, then $\Psi_{m} \varphi$ is invariant with respect to $\Gamma(1)$. As with the map $\Phi_{m}$, we have the stronger

Proposition 4. If $\varphi$ is a Maass wave form on $\Gamma(\sqrt{m})$ with eigenvalue $\lambda$, then either $\Psi_{m} \varphi$ is a Maass wave form on $\Gamma(1)$ with eigenvalue $\lambda$, or $\Psi_{m} \varphi \equiv 0$. Moreover, $\Psi_{m} \varphi$ is even if $\varphi$ is even, odd if $\varphi$ is odd.

Again, the proof is a direct verification. In this case, however, there is no analogue of Lemma 3: it is possible that $\varphi \not \equiv 0$, but $\Psi_{m} \varphi \equiv 0$. (The analogue 
of Lemma 3 would imply that $\Sigma(1)=\Sigma(\sqrt{2})=\Sigma(\sqrt{3})$, but Hejhal's calculation of the low end of each of these spectra rules out this possibility [3].)

\section{FOURIER EXPANSIONS AND HECKE OPERATORS}

A Maass wave form $f$ on $\Gamma(1)$ with eigenvalue $\lambda$ has the expansion

$$
f(z)=\sum_{n \neq 0} a_{n} W(|n| y) e^{2 \pi i n x}, \quad z=x+i y
$$

here, $W(y)=y^{1 / 2} K_{i r}(2 \pi y)$, with $r$ defined by the conditions $\lambda=\frac{1}{4}+r^{2}, r>0$, and $K_{i r}$ is the $K$-Bessel function [6,9]. Clearly, $f$ is even if and only if $a_{-n}=a_{n}$ in (4); $a_{-n}=-a_{n}$ is the condition for $f$ to be odd. For a Maass wave form $\varphi$ on $\Gamma(\sqrt{m})$ the expansion is

$$
\varphi(z)=\sum_{n \neq 0} \alpha_{n} W\left(\frac{|n| y}{\sqrt{m}}\right) e^{2 \pi i n x / \sqrt{m}} .
$$

Again, $\varphi$ is even or odd according as $\alpha_{-n}=\alpha_{n}$ or $\alpha_{-n}=-\alpha_{n}$, respectively.

Applying $\Phi_{m}$ to (4), we find that $\Phi_{2} f \equiv 0$ is equivalent to

$$
a_{n}+a_{2 n}=0, \quad a_{2 n+1}=0, \text { for all } n,
$$

and $\Phi_{3} f \equiv 0$ to

$$
a_{n}+a_{3 n}=0, \quad a_{3 n+1}=a_{3 n+2}=0, \text { for all } n .
$$

From these equations it follows directly that $\Phi_{m} f \equiv 0$ implies $f \equiv 0$, in either case. This provides a modified version of Lemma 3 .

The (weight- 0 ) Hecke operators for $\Gamma(1)$ are defined by

$$
T(p) f(z)=\frac{1}{p}\left\{f(p z)+\sum_{b=0}^{p-1} f\left(\frac{z+b}{p}\right)\right\}, \quad p \text { a prime. }
$$

Much less familiar are the Hecke operators for $\Gamma(\sqrt{2})$ and $\Gamma(\sqrt{3})$, introduced in [1]. For $p$ a prime (and again in weight 0 ), these are given by

$$
\begin{aligned}
T(m, p) \varphi(z) & =\frac{1}{p}\left\{\varphi(p z)+\sum_{b=0}^{p-1} \varphi\left(\frac{z+b \sqrt{m}}{p}\right)\right\}, \quad p \neq m, \\
& =\frac{1}{m}\left\{\varphi(m z)+\sum_{b=0}^{m-1} \varphi\left(\frac{z+b \sqrt{m}}{m}\right)+\sum_{b=1}^{m-1} \varphi\left(\frac{\sqrt{m} z+b}{\sqrt{m}}\right)\right\}, \\
& p=m .
\end{aligned}
$$

As is well known, the $T(p)$ preserve modular invariance (see, for example, [12, pp. 98-101]). Furthermore, a straightforward calculation with the hyperbolic Laplace operator shows that if $f$ is a Maass wave form on $\Gamma(1)$ with eigenvalue $\lambda$, then so is $T(p) f$. Similarly, the Hecke operators $T(m, p)$ preserve invariance with respect to the group $\Gamma(\sqrt{m})$ [1]. Again, if $\varphi$ is a Maass wave form on $\Gamma(\sqrt{m})$ with eigenvalue $\lambda$, then so is $T(m, p) \varphi$.

We summarize the action of the Hecke operators on the Fourier expansions. 
Suppose $f$ is a Maass wave form on $\Gamma(1)$, given by (4). Then

$$
T(p) f(z)=\sum_{n \neq 0} b_{n} W(n|y|) e^{2 \pi i n x}, \quad z=x+i y,
$$

where, for each prime $p$,

$$
b_{n}=a_{n p}+\frac{1}{p} a_{n / p}
$$

here, as usual, $a_{n / p}=0$ unless $p \mid n$. If $\varphi(z)$ is a Maass wave form on $\Gamma(\sqrt{m})$, given by (5), then

$$
T(m, p) \varphi(z)=\sum_{n \neq 0} \beta_{n} W\left(\frac{|n| y}{\sqrt{m}}\right) e^{2 \pi i n x / \sqrt{m}}, \quad z=x+i y,
$$

where

$$
\beta_{n}= \begin{cases}\alpha_{n p}+\frac{1}{p} \alpha_{n / p}, & \text { for prime } p \neq m, \\ \alpha_{n m}+\frac{1}{m} \alpha_{n / m}+\left(\delta_{n}-\frac{1}{m}\right) \alpha_{n}, & \text { for } p=m\end{cases}
$$

here,

$$
\delta_{n}= \begin{cases}1 & \text { if } m \mid n \\ 0 & \text { if } m \nmid n .\end{cases}
$$

Necessary and sufficient conditions for $f$ to be a Hecke eigenform on $\Gamma(1)$ are obtainable directly from (8). Similarly, (9) gives necessary and sufficient conditions for $\varphi$ to be a Hecke eigenform on $\Gamma(\sqrt{m})$.

We raise a question suggested by the remarks at the end of $\S 4$ : under what conditions does a given nontrivial Maass wave form on $\Gamma(\sqrt{m})$ lie in the kernel of $\Psi_{m}$ ? Invoking the expansion (5), we find that

$$
\left(\Psi_{m} \varphi\right)(z)=\sum_{n \neq 0}\left(\alpha_{n}+m \alpha_{m n}\right) W(|n| y) e^{2 \pi i n x} .
$$

In analogy to our observation concerning $\Phi_{m} f$, the identity $\Psi_{m} \varphi \equiv 0$ is equivalent to $a_{n}+m a_{m n}=0$, for all $n$. If $\Psi_{m} \varphi$ is a "Hecke eigenform" on $\Gamma(1)$, i.e., an eigenfunction of all the Hecke operators on $\Gamma(1)$ (always the case if, as conjectured, the space of Maass wave forms associated with a fixed eigenvalue has dimension 1), then $\Psi_{m} \varphi \equiv 0$ if and only if the coefficient in the term $n=1$ of (10) is zero, that is, $\alpha_{1}+m \alpha_{m}=0$.

Applying the same idea to $\varphi$ itself, we may normalize $\varphi$ so that $\alpha_{1}=1$. (Assuming that $\varphi \equiv 0$ is a Hecke eigenform on $\Gamma(\sqrt{m})$ implies $\alpha_{1} \neq 0$.) Then, $\varphi \not \equiv 0$ given by (5) satisfies $\Psi_{m} \varphi \equiv 0$ if and only if

$$
\alpha_{m}=-1 / m \text {. }
$$

Clearly, (11) can serve, as a crude check on any scheme for computing the coefficients $\alpha_{n}$ in (5), since for those Maass wave forms $\varphi \not \equiv 0$ on $\Gamma(\sqrt{m})$ associated with eigenvalues not in $\Sigma(1), \Psi_{m} \varphi \equiv 0$ and (11) holds of necessity.

\section{CONNECTIONS AMONG THE OPERATORS}

As a public service, I here list the results of a number of tedious calculations, omitting the calculations themselves. In each instance $f$ is a function of period 1 , and $\varphi$ is a function of period $\sqrt{m}$. In particular, the formulae apply to Maass wave forms $f$, on $\Gamma(1)$, and $\varphi$, on $\Gamma(\sqrt{m})$. 
We have:

$$
\begin{aligned}
& \left(\Psi_{m} \circ \Phi_{m}\right) f=(m+1) f+m T(m) f \\
& \left(\Phi_{m} \circ \Psi_{m}\right) \varphi=2 \varphi+m T(m, m) \varphi
\end{aligned}
$$

(a) $\left(\Phi_{m} \circ T(p)\right) f=\left(T(m, p) \circ \Phi_{m}\right) f, \quad p$ a prime $\neq m$,

(b) $\quad\left(\boldsymbol{\Phi}_{m} \circ T(m)\right) f+\frac{m-1}{m} \boldsymbol{\Phi}_{m} f=\left(T(m, m) \circ \Phi_{m}\right) f$;

(a) $\left(\Psi_{m} \circ T(m, p)\right) \varphi=\left(T(p) \circ \Psi_{m}\right), \quad p$ a prime $\neq m$,

(b) $\left(\Psi_{m} \circ T(m, m)\right) \varphi=\left(T(m) \circ \Psi_{m}\right) \varphi+\frac{m-1}{m} \Psi_{m} f$.

From (14) and (15) we infer:

(i) if $f$ is a Hecke eigenform (H.e.f.) on $\Gamma(1)$, then $\Phi_{m} f$ is H.e.f. on $\Gamma(\sqrt{m})$

(ii) $\varphi$ H.e.f. on $\Gamma(\sqrt{m})$ implies $\Psi_{m} f$ is H.e.f. on $\Gamma(1)$.

Furthermore, since $\Phi_{m}$ has a trivial kernel, it follows that if $\Phi_{m} f$ is H.e.f. on $\Gamma(\sqrt{m})$, then $f$ is H.e.f. on $\Gamma(1)$. By way of contrast, because $\Psi_{m}$ has a nontrivial kernel, we cannot infer that $\varphi$ is H.e.f. on $\Gamma(\sqrt{m})$ from the assumption that $\Psi_{m} \varphi$ is H.e.f. on $\Gamma(1)$.

\section{CONCLUding REMARKS}

As with the Hecke operators on $\Gamma(1)$, the Hecke operators on $\Gamma(\sqrt{m})$ need not be restricted to prime index; they can, in fact be defined for each $l \in Z^{+}$. Since all $T(m, l)$ are expressible as polynomials in the $T(m, p) \quad\left(l \in Z^{+}, p\right.$ a prime), $\varphi$ is an eigenfunction for all $T(m, l)$ if it is an eigenfunction for the $T(m, p)$. The definition of $T(m, l)$ can be extended to weights other than 0 $[1]$.

\section{BIBLIOGRAPHY}

1. J. Bogo and W. Kuyk, The Hecke correspondences for $\overline{G(\sqrt{q})}, q$ prime; Eisenstein series and modular invariants, J. Algebra 43 (1976), 585-605.

2. E. Hecke, Lectures on Dirichlet series, modular functions and quadratic forms, Edwards Bros., Inc., Ann Arbor, 1938.

3. D. Hejhal, Eigenvalues of the Laplacian for Hecke triangle groups, Mem. Amer. Math. Soc., No. 469, Providence, RI, 1992.

4. _ Eigenvalues of the Laplacian for $\operatorname{PSL}(2, Z)$ : some new results and computational techniques, International Symposium in Memory of Hua Loo-Keng (S. Gong, Q. Lu, Y. Wang, and L. Yang, eds.), vol. 1, Science Press and Springer-Verlag, 1991, pp. 59-102.

5. D. Hejhal and S. Arno, On Fourier coefficients of Maass waveforms for $\operatorname{PSL}(2, Z)$, Math. Comp. 61 (1993), 245-267.

6. H. Maass, Die Differentialgleichungen in der Theorie der elliptischen Modulfunktionen, Math. Ann. 125 (1953), 235-263.

7. __ Über eine neue Art von nichtanalytischen automorphen Funktionen und die Bestimmung Dirichletscher Reihen durch Funktionalgleichungen, Math. Ann. 121 (1949), 141-183.

8. O. Rausenberger, Zur Theorie der Funktionen mit mehreren, nicht vertauschbaren Perioden, Math. Ann. 20 (1882), 47-48.

9. W. Roelcke, Das Eigenwertproblem der automorphen Formen in der hyperbolischen Ebene, I, Math. Ann. 167 (1966), 292-337. 
10. A. Selberg, Harmonic analysis and discontinuous groups in weakly symmetric Riemannian spaces with applications to Dirichlet series, J. Indian Math. Soc. 20 (1956), 47-87.

11. _ On the estimation of Fourier coefficients of modular forms, Proc. Sympos. Pure Math., vol. 8, Amer. Math. Soc., Providence, RI, 1965, pp. 1-15.

12. J.-P. Serre, A course in arithmetic, Springer-Verlag, Berlin and New York, 1973.

13. H. Stark, Fourier coefficients of Maass waveforms, Modular forms (R.A. Rankin, ed.), Halsted Press, New York, 1984.

14. A. Winkler, Cusp forms and Hecke groups, J. Reine Angew. Math. 386 (1988), 187-204.

Department of Mathematics, Temple University, Philadelphia, Pennsylvania 19122 